RASĀYAN J. Chem.

Vol. 14 | No. 2 |698-704| April - June | 2021 ISSN: 0974-1496 | e-ISSN: 0976-0083 | CODEN: RJCABP http://www.rasayanjournal.com http://www.rasayanjournal.co.in

\title{
THE OPTIMIZATION OF $\alpha$ - MANGOSTIN AS A NEW DRUG CANDIDATE THROUGH MOLECULAR DOCKING AND DYNAMIC SIMULATIONS
}

\author{
D. R. Pomalingo, C. Suhandi, S. Megantara and M. Muchtaridi ${ }^{\bowtie}$ \\ Department of Pharmaceutical Analysis and Medicinal Chemistry, Faculty of Pharmacy, \\ Padjadjaran University, 45363 Sumedang, Indonesia. Telephone / Fax: (022) 84288888 ext. 3210 \\ Corresponding Author: muchtaridi@unpad.ac.id
}

\begin{abstract}
$\alpha$-mangostin has a strong pharmacological effect in both in vitro and in vivo testing. It is examined by targeting several cellular targets with various mechanisms of action. Various approaches have been made to determine the efficacy of $\alpha$-mangostin. One of them is a molecular modelling technique in which it alters the structure of a compound using the computational technique. This well-known technique is conducted through studies of molecular docking and Molecular dynamics as a part of new drug development. This review study aimed to show the role of the application of the molecular modelling approach of $\alpha$-mangostin as a new drug candidate.
\end{abstract}

Keyword: $\alpha$ - Mangosteen, Molecular Docking, Molecular Dynamics, Drug Candidate

RASĀYAN J. Chem., Vol. 14, No.2, 2021

\section{INTRODUCTION}

Mangosteen (Garcinia mangostana) is a fruit grown in tropical regions, such as Southeast Asia, India, and Sri Lanka. The pericarp (skin) of this fruit has been used empirically from time to time as a traditional medicine for some diseases treatment. ${ }^{1}$

Ever since ancient times, mangosteen has been known as hereditary medicine to cure chronic diarrhea, wound infections, skin infections, and dysentery. Several studies have been proved that mangosteen pericarp has a major compound of xanthone derivate that is $\alpha$-mangostin This xanthone derivative has demonstrated a few pharmacological activities such as antifungal, antioxidant, antibacterial ${ }^{2}$, anti-tumor, anti-inflammatory, and anti-allergic. , $^{1,3,4}$

Besides having pharmacological properties, $\alpha$-mangostin also has several disadvantages such as low solubility and low bioavailability. Various studies have been conducted to determine the effects of $\alpha$ mangostin both in vitro, in vivo and silico. This review explains the Molecular dynamic simulations and Molecular Docking as assisting $\alpha$-mangostin as an inhibitor of some diseases.

\section{Molecular Docking and Dynamic Simulations of $\alpha$-Mangostin} $\alpha$-Mangostin as Inhibitor $\alpha / \beta$-Amyloid Aggregation

Alzheimer, a neurodegenerative disease, is caused by the plaque in the intraneuronal neurofibrillary tangles, neurons and synapses degradation and extracellular senile in the brains of a person with the disease. The $\beta$-amyloid peptides $(\alpha \beta)$ consist 39-43 residual amino acids is the main constituent of amyloid plaques that is crucial to the neuropathogenesis of Alzheimer's disease. ${ }^{5}$ A structure of $\alpha \beta / \beta$ Amyloid Aggregation was taken from Protein Data Bank (PDB) with ID: 1BA4. ${ }^{6}$ The structure of $\alpha$ mangostin, novel breast cancer drug, was docked to $\alpha \beta$-amyloid. $\alpha$-mangostin as the potential molecule comprising antagonist properties toward $\mathrm{hER} \alpha . \alpha$-mangostin is the active secondary metabolite primarily derived from the pericarp part of Garcinia mangostana containing antiproliferative properties associated with suppression of tumor growth; it inhibits the proliferation of MCF-7 cell lines through inhibiting of $\mathrm{hER} \alpha$ activity. ${ }^{7}$ Anti-proliferative activity of $\alpha$-mangostin against MCF-7 cell lines observed with $\mathrm{IC}_{50}$ by $20 \mu \mathrm{g} / \mathrm{mL} .{ }^{8}$ The $\mathrm{IC}_{50}$ value observed was categorized in the less active category ranging from 10 to $100 \mu \mathrm{g} / \mathrm{Ml} .{ }^{9}$ Thus, this study was performed to modify the $\alpha$-mangostin as a parent structure and investigated its molecular interactions to enhance its antagonistic activity toward $\mathrm{hER} \alpha$ through molecular 
RASĀYAN J. Chem.

Vol. 14 | No. 2 |698-704| April - June | 2021

docking and molecular dynamics simulation utilizing MOE's (Molecular Operating Environment) automatic docking algorithm was used. The top thirty poses from $\alpha$-mangostin were determined over the conformational search, and further the all poses were docked into a receptor where the grid box was set due to binding site evaluation and energy minimization. The binding mode result was determined from the superior ligand pose. Based on the results, it has been found the potential energy and interactions between ligand and receptor. MOE was employed in this study that gives hydrophobic surfaces and a diagram of the interaction between the ligands and the receptors. Molecular Dynamics (MD) of complex $\alpha$-mangostin and $\alpha \beta$ helix was achieved at $20 \mathrm{~ns}$ using the Amber v.11on GPU-based workstations produced by Hewlett-Packard, USA. The general model in macromolecular simulations is preferred to water. During the simulation, the temperature was set at $300 \mathrm{~K}$ and the cut-off distance was set at $12 \AA$. During the minimization phase and simulation, the ff99SB force field was set as the force field for this simulation. As polyphenols, $\alpha$-mangostin has a similar effect to curcumin and resveratrol as in neuroprotective effects by leading the conformation of $\beta$-helix and a transition conformation between $\alpha$ helix and $\beta$-sheets. ${ }^{7}$ Thus, it retains $\alpha \beta$ on a conformational $\alpha$-helix. Firstly, the molecular docking analysis is performed in the PDB complex of 1BA4, 13-40 residues were obtained and conservatively estimated form with $\alpha$-helix. Therefore, the helix $\alpha \beta$ includes $13-40$ residues were used in docking analysis.

$\alpha$-mangostin interacts with the helix $\alpha \beta$ through the hydrophobic surface. The phenolic hydroxyl group $\alpha$ mangostin forms a hydrogen bond with some amino acid residue (Lys16 and Asp23 of $\alpha \beta$ with bond length $2.68 \AA$ and $2.29 \AA)$. Phe 19 of $\alpha \beta$ has pi-pi cation interaction $(3,45 \AA)$ with the aromatic ring of $\alpha$ mangostin. This molecule interacts with Glu22 of $\alpha \beta$. The binding energy of $\alpha$-mangosteen with the $\alpha$ helix conformation of $\alpha \beta$ is $-68.76 \mathrm{kcal} / \mathrm{mol}$. Molecular docking result provides information about molecular interactions and direct interactions between $\alpha$-mangostin and $\alpha \beta$-amyloid at the level of atomic. To learn more about the $\alpha$-mangostin function in the interaction, MD simulations were performed. RMSD of $\alpha \beta$ helix spine is calculated to show the entire process of conformation changes in the helix. The RMSD plot shows severe conformation variations in a short period. ${ }^{8}$

RMSD for $\alpha \beta$ helix is highly fluctuating. The fluctuation of the RMSD plot starts within $3 \AA$ until $8 \AA$ for $7 \mathrm{~ns}$, and also within $6 \AA$ until $10 \AA$ for $13 \mathrm{~ns}$. It showed that the changes of conformation of helical $\alpha \beta$ are still in a steady-state or unstable for $20 \mathrm{~ns}$ of MD simulation. As for the $\alpha$-mangostin and $\alpha \beta$ helix complex, RMSD starts at $3 \AA$ and reaches the equilibrium state at $8 \AA$, and $7 \mathrm{~ns}$ after MD simulation. The softer plot of the complex shows that the complex has a more stable conformation than helical $\alpha \beta$. On the other hand, the hydrogen bond analysis of the simulated pathway of MD shows that hydrogen bonds are stable at $\alpha$-mangostin and Glu22 (Glutamine), also Asp23 and helical $\alpha \beta$. MD simulation and molecular docking result showed that $\alpha$-mangostin binds to $\alpha \beta$ strongly, which shows stabilized conformations, indicating that $\alpha$-mangostin may disrupt with $\alpha \beta$ aggregation. 3D structure of fibrils $\alpha \beta$ (PDB ID: $2 \mathrm{BEG}$ ) showed before ${ }^{9}$. $\alpha \beta$ represents a-b-strand turn into b-strand consisting of two intermolecular, $b$-sheet registers by 18-26 residual amino acids (b1) and 31-42 residual amino acids (b2. The structure-activity relationships study shows that every efficient polyphenol inhibitor at least consists of two phenolic rings containing at least two to six atomic connectors and three hydroxyl groups in an aromatic ring. It is central to the inhibition of fibrils by strengthening hydrophobic interactions between aromatic rings with a $\beta$-sheet structure by hydrogen bonding. ${ }^{10}$ According to the molecular docking results in the study, the corresponding form of two molecules makes the $\alpha$-mangostin feasible to fall into $\alpha \beta$ binding site, hydrophobic interactions between Phe19 amino acid residue of $\alpha \beta$ and $\alpha$-mangostin aromatic ring, and enhancing the formation of hydrogen bond interactions between amino acid residues of Lys16, Glu22, and Asp 23 of the hydroxyl group $\alpha \beta$ and phenolics of $\alpha$-mangostin. It can be seen that $\alpha$-mangostin is also polyphenolic inhibitor. The RMSD value of the MD simulation is generally used as an indicator of the conformation variation from the structure to the equilibrium phase in comparison to the library confirmation. ${ }^{11}$ The MD simulation further demonstrates a smooth movement of RMSD change from helical $\alpha \beta$ when the complex compounds with $\alpha$-mangostin as compared to $\alpha \beta$ alone, thereby indicating that $\alpha$-mangostin is attached to the $\alpha \beta$ helix as expected, resulting in the more stable conformation of the $\alpha \beta$. There is stable hydrogen bond interactions between Glu22, Asp23, and $\alpha$-mangostin. The hydrogen bond between $\alpha$-mangostin and Lys 16 that is absent in MD simulations can persist, thus stabilizing helical 
RASĀYAN J. Chem.

Vol. 14 | No. 2 |698-704| April - June | 2021

$\alpha \beta$, may be short-lived as the hydrogen bonds examined in the MD simulation should be at least $5 \%$ of the total life span. Natrium or Sodium connecting Asp23 and Lys28 is crucial to the process of fibrils $\alpha \beta$ growth. Blocking the salt bridge formation stabilizes the helical $\alpha \beta$ and restrains the fiber growth process of $\alpha \beta .^{12}$ Despite some differences, the docking result is validated by these data. The hydrogen bond between $\alpha$-mangostin and Asp23 can inhibits the sodium bridge between Asp23 and Lys28. Another interaction among helical $\alpha \beta$ and $\alpha$-mangostin can stabilize the complex. Thus the researcher hypothesizes that the binding of $\alpha$-mangostin to $\alpha \beta$ ends up in the lack of nucleation competent core formation, thereby inhibiting and separating aggregation of $\alpha \beta$. Still, in silico energy docking and MD simulation results are only of the estimated activity indicators, hence it needs further research to explain more precisely the mechanism. $\alpha$-mangostin is generally acknowledged as an effectual antioxidant and ROS scavenger. ${ }^{13}$ Although the researchers demonstrate their anti-amyloidogenic properties, the anti-oxidants effects of $\alpha$ mangostin cannot be completely excluded in their neuroprotective properties against oligomers $\alpha \beta$. Nonetheless, $\alpha$-mangostin shows ROS flushing properties in the level of micromolar ${ }^{4,14}$, which is much higher than the molar nanoscale concentration used in the present study. On top of that, undetectable oxidative species in the neurons dealing with oligomers $\alpha \beta$ (data are not provided) that resonates with the previous studies show that oxidative stress properties of the $\alpha \beta$ are not involved in its oligomeric cytotoxicity of $\alpha \beta{ }^{15}$ Therefore, the effect of $\alpha$-mangostin anti-neurotoxicity on $\alpha \beta$ oligomers significantly depends on the direct dissociation of oligomers $\alpha \beta$. In this research, it is apparent that $\alpha$-mangostin has strong protection against the neurotoxicity caused by $\alpha \beta$ aggregates as a toxic form. The involving mechanism can be linked to its ability in redirecting the toxic $\alpha \beta$ cascade by separating its oligomers accumulated with the $\beta$-sheet-rich and fibrils of the later stages, and the fibril formation inhibition. $\alpha$ Mangostin is a fat-soluble molecule $(<600 \mathrm{Da})$ with a possibility to pass the barrier of the central nervous system, ${ }^{16}$ thus it holds potential as a treatment for patients with Alzheimer's disease.

\section{$\alpha$-Mangostin against MRSA As An Outcome of Membrane Targeting}

The molecular interaction model of the $\alpha$-mangostin and the model of the bacterial membrane using water as solvent water was simulated into the molecular docking and examined using molecular dynamics (MD). Even though the composition of lipids from different bacterial membranes varies, the main component of the membrane is POPE (1-Palmitoyl-2-oleoyl-sn-glycerol-3-phosphoethanolamine) and POPG (1-palmitoyl-2-oleoyl -n- glycerol-3-phosphoglycerol). ${ }^{17}$ As result, this study uses 128 mixed lipids with a scale of POPG : POPE in a comparison of $3: 1$ to depict and draw a common membrane model ${ }^{17}$. Lipid molecules and $\alpha$-mangostin is model using the strengths column of Gromos53a6 ${ }^{18,20}$, while solvent-soluble molecules use the SPC model. ${ }^{21}$ The parameters of $\alpha$-mangostin are made by the Topology Builder column (ATB). ${ }^{22}$ Two simulations using a molecule of $\alpha$-mangostin, similar $\alpha$ mangostin in high concentration. Within those simulations, a randomized $\alpha$-mangostin molecule is attached to the membrane model of the bacteria. Subsequently, the system is dissolved with 7200 molecules of water and neutralized with sodium ions. Before MD simulation production phase, the system is composed of 500 times minimizing energy by employing steep linear algorithms, set up at 10 NVT simulations. The simulation of MD ns 250 can be finished in that case, which corresponds to different $\alpha$-mangostin concentrations. To add a conformation sample of $\alpha$-mongostin at a specific range from the center of the bilayer, a series of determining distance simulations was performed by overcoming the distance between a bilayer center and an $\alpha$-mangostin; the process relied on a program named gromacs pull module. During all the process of MD simulations, $1.2 \mathrm{~nm}$ distance was utilized for two kind interactions consist LJ and real space electrostatic, and the algorithm of Ewald-particle mesh was utilized for calculating the interactions of long-distance electrostatic in the repeated space. The method of Nose-Hoover was applied to keep the target temperature at $310^{\circ} \mathrm{K}$. The Parrinello-Rahman method with a mode of semi-isotropic coupling was then applied in keeping up the pressure at $1 \mathrm{~atm}$ in the gamma NPT. ${ }^{23}$ To understand membrane disturbance at the atomic level, the author uses MD simulation to provide information about $\alpha$-mangostin membrane penetration pathways using bacterial membrane models. They determine the penetration of $\alpha$-mangostin into POPE / POPG (75/25) bilayers using simulations of all MD atoms. The observation of the absorption of molecules in the membrane was performed once $\alpha$-mangostin was placed in the membrane. In the ratio of low drug/lipid (1/128). ${ }^{24}$ 
RASĀYAN J. Chem.

Vol. 14 | No. 2 |698-704| April - June | 2021

The distance between lipid bilayers and $\alpha$-mangostin is changed to $100 \mathrm{~ns}$ but reaches $1.5 \mathrm{~nm}$ equilibrium, which indicates that $\alpha$-mangostin could get toward lipid bilayer immediately, located under the group of lipid molecule heads. In addition to $\alpha$-mangostin molecules, some $\alpha$-mangostins penetrate the bacterial membrane at the lower region that is modeled above the simulation. ${ }^{24}$

It is accompanied by a disorder of the membrane-defective integrity (filled with water molecules) because of $\alpha$-mangostin's hydrophobic interaction and bacterial membrane enhances the permeability of bacteria lipid membrane and also helps the penetration process of $\alpha$-mangostin. Moreover, it also accommodates hydrophobic interactions between $\alpha$-mangostin and lipid-tails part of phospholipid since $\alpha$-mangostin can reach lower leaflets of lipid bilayers. The observation indicates that membrane perturbation performed by the $\alpha$-mangostin tends to amplify biophysical studies data and SEM results reported. ${ }^{24}$

The research showed that $\alpha$-mangostin is at a different range from the center of the bilayer. In the process of penetration, the isoprenyl group, driven by hydrophobic interactions, incorporates the hydrophobic area of the membrane. After that $\alpha$-mangostin is induced by the entropy as the long axis is swirled with lipid tail. $^{13}$

Molecular dynamics simulation shows that a strong $\alpha$-mangostin hydrophobic association with an alkyl lipid chain is a powerful boosting for $\alpha$-mangostin's penetration. The isoprenyl group is bound to xanthone structure as a short lipid tail that is found, thus triggering the penetration toward hydrophobic area of the membrane. Specifically, it could be stated that the isoprenyl group has a function to lower the free energy barrier from penetration. On top of that, the presence of the isoprenyl group improves hydrophobicity of $\alpha$-mangostin, thereby increasing the possibility to split into membranes. Altogether, the isoprenyl groups produce stronger antibacterial properties. The result shows that hydrophobicity of $\alpha$ mangostin is more soluble by hydrophobic lipids. Less than one isoprenyl group contained in SZ-1 region and SZ-5 region has lower anti-microbial activities as a free energy barrier to the penetration of great bacterial membranes. The results also correspond to the new reports which indicate that at least two isoprenyl groups that conjugate with xanthone scaffolding are essential for conducting strong antimicrobial properties. ${ }^{1,2}$ Besides, during the simulation, it is found many water defects. Regardless of the inactivity of some defects, some disabilities develop into the translocation of water through the membrane. In the long-term, higher permeability can also cause large molecules to spread across membranes that cause intracellular components leakage. It is certain that a forceful $\alpha$-mangostin affinity for the hydrophobic chain in the alkyl group, produces packing density and integrity, thus forming intracellular components leakage. MD simulations and biophysical studies reveal a rapid disruption of the integrity of internal membrane bacteria as the main rider of the cell even though $\alpha$-mangostin is renowned for its interaction with the precursor of transmembrane proteins through hydrogen bonding. ${ }^{8} \mathrm{An}$ observation should be taken regarding the coefficients of the $\alpha$-mangostin partition using the HighPerformance Liquid Chromatography (HPLC) method following guidelines of OECD for chemical testing ${ }^{25}$ in supporting the role of $\alpha$-mangostin hydrophobic interaction in the membrane of bacteria. The $\log P$ value of the given $\alpha$-mangostin is 6.4 , signifying a strong hydrophobic property of $\alpha$-mangostin and explaining the strong tendency to split into bacterial membranes through hydrophobic interactions. On that ground, the current data draws that hydrophobic interaction is very important for harmful bacteria. In conclusion, this study shows that bacterial membrane layers are the main target for $\alpha$-mangostin activity towards Gram-positive bacterials. $\alpha$-Mangostin is straightforwardly extracted from common tropical fruits. The indications of $\alpha$-mangostin cause membrane disorders and intracellular content leakage in five to ten minutes. The $\alpha$-mangostin hydrophobic associations with lipid membranes cause membrane deformation and water molecule diffusion across the membrane. On the other hand, the resistance is not created in laboratory simulations utilizing MRSA and E. faecalis. Further, $\alpha$-mangostin is more effective against bacterial cell membranes rather than mammalian cell membranes in MIC and bacterial concentrations. These outcomes claim that $\alpha$-mangostin, as a basic-structure molecule, is a potential object for further studies and development.

\section{$\alpha$-Mangostin against Estrogen As An Outcome of Breast Cancer Targeted}

Of the common cancers among women in Asia is breast cancer. The prevalence of breast cancer has seen a steady rise over the past decades. ${ }^{26}$ Based on data GLOBOCAN (IARC) in 2012, cancer causes the 
RASĀYAN J. Chem.

Vol. 14 | No. 2 |698-704| April - June | 2021

death of about 8.2 million people. Cancer rates are expected to increase annually and, by 2030, are expected to reach 23.6 million new cases per year. ${ }^{27}$ As based on several experiments and clinical and epidemiological evidence, the development and progression of breast cancer are heavily determined by ovarian hormones; these hormones are central to the normal breast tissues' growth and differentiation. ${ }^{28}$ Estrogens function in enhancing the growth in estrogen-responsive target tissues, including the breast. For this reason, estrogens are the risk factor for developing cancer. Studies have examined different nonsteroidal compounds other than estradiol (the natural ligand, including tamoxifen $;{ }^{29}$ the results reveal that the compounds have different effects as agonists or antagonists; this is determined by the particular organ system or gene. ${ }^{30} \mathrm{ER} \alpha$ is a ligand-activated transcription factor; its functions are crucial to many tissues and it is also significant to the etiology of breast cancer. ${ }^{31,32}$ Considering that ER $\alpha$ is essential for treating and preventing breast cancer, numerous molecules have been designed to bind ER $\alpha$ and evoke distinct pharmacological profiles. Hence, it is essential to examine compounds of natural products that serve as antagonists of estrogen in breast tissue and as agonists in bone and cardiovascular tissues. ${ }^{3}$ Other than estradiol (the natural ligand), various nonsteroidal compounds, including tamoxifen ${ }^{29}$, have been studied. The compounds have different effects as agonists or antagonists; it depends on the particular organ system or gene. ${ }^{30}$

$\mathrm{ER} \alpha$ refers to a ligand-activated transcription factor with key functions to many tissues and the etiology of breast cancer. ${ }^{31,32}$ Various molecules have been designed to bind ER $\alpha$ and elicit distinct pharmacological profiles given that ER $\alpha$ is essential in the treatment and prevention of breast cancer. Hence, it is of paramount importance to identify compounds of natural products that act as antagonists of estrogen in breast tissue and as agonists in bone and cardiovascular tissues. ${ }^{3}$

In the antagonist mechanism of estrogen, the restrained Helix-11 would forceHelix-12 to be set in the "mousetrap" like no other preferable (antagonist) conformation is found on the surface of the hER $\alpha$ ligand-binding site. The network zipping $\mathrm{H} 3$ and $\mathrm{H} 11$ in estrogen stabilized conformation of agonists. This zipper network proves that H12 is set in the "mousetrap", and consequently, it is unable to get into the antagonist position on the hER LBD surface when H11 is prohibited to relax. However, this network zipper is lost once an antagonist (4-OHT) is placed ${ }^{33}$. Mechanism of natural product compounds against human estrogen is studied by Muchtaridi et al. ${ }^{13,33,35}$ As anti-breast cancer, $\alpha$-mangostin serves as an antiproliferative associated with inhibiting the growth of breast cancer cells MCF-7 through a reduced function of $\mathrm{hER} \alpha$ receptors (most common breast cancer subtype).$^{36}$ Apoptosis of cancer cells is induced by $\alpha$-mangostin through mitochondrial pathways and Aktdephosphorylation on the breast cancer cell. $\alpha$ mangostin inhibits invasion also an exodus of cancer cells in the breast gland. MCF-7 adenocarcinoma cell apoptosis is inhibited by $\alpha$-mangostin thus it shows as an anti-proliferative activity with an $\mathrm{IC}_{50}$ value of $20 \mathrm{Mm}^{37,38}$

It is essential to examine the molecular interactions and the pharmacophore-fit of alpha mangostin and its derivatives with estrogen receptor $\alpha(\mathrm{ER} \alpha)$ using molecular docking simulation and pharmacophore modeling approaches. This is to find out the new anti-breast cancer. ${ }^{39}$

\section{CONCLUSION}

Mangosteen has been used for the treatment of many diseases empirically from year to year. Besides, much research proves that the compound from mangosteen called $\alpha$-mangostin is effective in use for disease treatment in animal testing. The structure $\alpha$-mangostin that has phenolic rings, proves that $\alpha-$ mangostin could penetrate the lipid membrane of bacteria. Furthermore, all of the different possesses of $\alpha$-mangostin in molecular modeling is a potential candidate for a new drug. In the end, it is expected that this research can open up new avenues to find and design a new drug candidate or contributes to further development and studies.

\section{ACKNOWLEDGMENT}

This study was supported by a Master degree in Pharmacy, Universitas Padjadjaran of Indonesia.

\section{REFERENCES}

1. G. Chen, Y. Li, W. Wang, and L. Deng, Expert Opinion On Therapeutic Patents, 28(5), 415(2018), DOI: $10.1080 / 13543776.2018 .1455829$ 
RASĀYAN J. Chem.

Vol. 14 | No. 2 |698-704| April - June | 2021

2. G.M. Cragg, D.J. Newman, and K.M. Snader. Journal of Natural Products, 60(1), 52(1997), DOI: $10.1021 / \mathrm{np} 9604893$

3. H.R.W. Dharmaratne, Y. Sakagami, K.G.P. Piyasena, and V. Thevanesam, Natural Product Research, 27(10), 938(2013), DOI:10.1080/14786419.2012.678348

4. J. Pedraza-Chaverrí, L.M. Reyes-Fermín, E.G. Nolasco-Amaya, M. Orozco-Ibarra, O.N. MedinaCampos, O. González-Cuahutencos, and R. Mata, Experimental and Toxicologic Pathology, 61(5), 491(2009), DOI:10.1016/j.etp.2008.11.002

5. L. Crews, and E. Masliah, Human Molecular Genetics, 19(R1), R12(2010), DOI: $10.1093 / \mathrm{hmg} / \mathrm{ddq} 160$

6. M. Coles, W. Bicknell, A.A. Watson, D.P. Fairlie, and D. Craik, Journal Biochemistry, 37(31), 11064(1998), DOI:10.1021/bi972979f

7. P. Deepa, and M. Jayakannan, Journal of Polymer Science Part A: Polymer Chemistry, 46(7), 2445(2008), DOI:10.1073/pnas.0711731105

8. Y. Wang, Z. Xia, J.R. Xu, Y.X. Wang, L.N. Hou, Y. Qiu, and H.Z. Chen, Neuropharmacology, 62(2), 871(2012), DOI:10.1016/j.neuropharm.2011.09.016

9. J.A. Lemkul, and D.R. Bevan, Biochemistry, 49(18), 3935(2010), DOI:10.1021/bi1000855

10. F. Re, C. Airoldi, C. Zona, M. Masserini, B.L. Ferla, N. Quattrocchi, and F. Nicotra, Current Medicinal Chemistry, 17(27), 2990(2010), DOI:10.2174/092986710791959729

11. Y. Wang, Z. Xia, J.R. Xu, Y.X. Wang, L.N. Hou, Y. Qiu, and H.Z. Chen, Neuropharmacology, 62(2), 871(2012), DOI:10.1016/j.neuropharm.2011.09.016

12. Huet, Alexis, and Philippe Derreumaux, Biophysical Journal, 91(10), 3829(2006), DOI:10.1529/biophysj.106.090993

13. Swamy, Mallappa, and Uma Sinniah, Molecules, 20(5), 8521(2015), DOI: $10.3390 /$ molecules 20058521

14. Y. Sánchez-Pérez, R. Morales-Bárcenas, C.M. García-Cuellar, R. López-Marure, M. CalderonOliver, J. Pedraza-Chaverri, and Y.I. Chirino, Chemico-biological Interactions, 188(1), 144(2010), DOI: $10.1016 /$ j.cbi.2010.06.014

15. A. Boldyrev, A. Koudinov, T. Berezov, and D.O. Carpenter, Journal of Alzheimer's Disease, 6(6), 633(2004), DOI: 10.3233/jad-2004-6607

16. Porat, Yair, Adel Abramowitz, and Ehud Gazit, Chemical Biology and Drug Design, 67(1), 27(2006), DOI:10.1111/j.1747-0285.2005.00318

17. W. Zhao, T. Róg, A.A. Gurtovenko, I. Vattulainen, and M. Karttunen, Biochimie 90(6), 930(2008), DOI: $10.1016 /$ j.biochi.2008.02.025

18. C. Oostenbrink, A. Villa, A.E. Mark, and W.F. Van Gunsteren, Journal of Computational Chemistry, 25(13), 1656(2004), DOI:10.1002/jcc.20090

19. Jehle, Herbert. Proceedings of the National Academy of Sciences of the United States of America, 50(3), 516(1963), DOI: 10.1073/pnas.50.3.516

20. Terzyk, and P. Artur, Journal of Colloid and Interface Science, 275(1), 9(2004), DOI: 10.1016/j.jcis.2004.02.011

21. Buckingham, Amyand David, Philosophical Transactions of the Royal Society of London. B, Biological Sciences, 272(915), 5(1975), DOI:10.1098/rstb.1975.0066

22. A.K. Malde, L. Zuo, M. Breeze, M. Stroet, D. Poger, and P.C. Nair, Journal of Chemical Theory and Computation, 7(12), 4026(2011), DOI:10.1021/ct200196m

23. P. Bjelkmar, P. Larsson, M.A. Cuendet, B. Hess, and E. Lindahl, Journal of Chemical Theory and Computation 6(2), 459(2010), DOI:10.1021/ct900549r

24. J.J. Koh, S. Qiu, H. Zou, R. Lakshminarayanan, J. Li, and X. Zhou, Biochimica et Biophysica Acta (BBA)-Biomembranes, 1828(2), 834(2013), DOI:10.1016/j.bbamem.2012.09.004

25. T. Lührs, C. Ritter, M. Adrian, D. Riek-Loher, B. Bohrmann, H. Döbeli, and R. Riek, Proceedings of the National Academy of Sciences, 102(48), 17342(2005), DOI:10.1073/pnas.0506723102

26. J.R. Harris, M.E. Lippman, U. Veronesi, W. Willett, New England Journal of Medicine, 327(5), 319(1992), DOI:10.1056/nejm199207303270505 
27. J. Ferlay, I. Soerjomataram, R. Dikshit, S. Eser, C. Mathers, M. Rebelo, and F. Bray, International Journal of Cancer, 136(5), E359(2015), DOI:10.1002/ijc.29210

28. Bernstein, Leslie, and F. Michael, JNCI: Journal of the National Cancer Institute, 90(1), 5(1998), DOI: $10.1093 /$ jnci/90.1.5

29. V. Jordan, Craig, and Monica Morrow. Endocrine Reviews, 20(3), 253(1999), DOI: $10.1210 /$ edrv.20.3.0368

30. M.J. Broeders, and A. L. Verbeek, The Quarterly Journal of Nuclear Medicine: Official Publication of the Italian Association of Nuclear Medicine (AIMN)[and] the International Association of Radiopharmacology (IAR), 41(3), 179(1997)

31. J.F. Couse, and K.S. Korach, Endocrine Reviews, 20(4), 459(1999), DOI:10.1210/edrv.20.3.0370

32. K. Hunt, International Journal of Fertility and Menopausal Studies, 39, 67(1994).

33. I. Musfiroh, M. Muchtaridi, A. Muhtadi, A. Diantini, A.N. Hasanah, L.Z. Udin, Y. Susilawati, R. Mustarichie, R.E. Kartasasmita, and S. Ibrahim, Journal of Applied Pharmaceutical Science, 3(6), 7(2013), DOI: 10.7324/JAPS.2013.3602

34. M. Muchtaridi, M. Yusuf, A. Diantini, S.B. Choi, B.O. Al-Najjar, J.V. Manurung, H.A. Wahab, International Journal of Molecular Sciences, 15(5), 7225(2014), DOI:10.3390/ijms15057225

35. M. Muchtaridi, H.N. Syahidah, A. Subarnas, M. Yusuf, S.D. Bryant, and T. Langer, Pharmaceuticals, 10(4), 81(2017), DOI: 10.3390/ph10040081

36. Y.S. Won, J.H. Lee, S.J. Kwon, J.Y. Kim, K.H. Park, M.K. Lee, K.I. Seo, Food and Chemical Toxicology, 66, 158(2014), DOI:10.1016/j.fct.2014.01.040

37. A. Setiawati, Indonesian Journal of Pharmacy, 25(3), 119(2014), DOI:10.14499/indonesianjpharm25iss3pp119

38. H. Kurose, M. Shibata, M. Iinuma, and Y. Otsuki, Journal of Biomedicine and Biotechnology, 2012, 1(2012), DOI: $10.1155 / 2012 / 672428$

39. M. Muchtaridi, D. Dermawan, and M. Yusuf. Journal of Young Pharmacists, 10(3), (2018), DOI: $10.5530 /$ jyp. 2018.10 .58

40. H. Kurose, M.A. Shibata, M. Iinuma, Y. Otsuki, BioMed Research International, (2012), Article ID 672428(2012), DOI:10.1155/2012/672428

41. T. Lührs, C. Ritter, M. Adrian, D. Riek-Loher, B. Bohrmann, H. Döbeli, D. Schubert, and R. Riek, Proceedings of the National Academy of Sciences, 102(48), 17342(2005). DOI: $10.1073 /$ pnas.0506723102

42. World Health Organization, Promoting Rational Use of Medicines: Core Components, No. WHO/EDM/2002.3, Geneva: World Health Organization, 2002.

43. Y. Sánchez-Pérez, R. Morales-Bárcenas, C.M. García-Ceullar, R. Lopéz-Marure, M. CalderonOliver, J. Pedraza-Chaverri, and Y.I. Chirino, Chemico-biological Interactions, 188(1), 144(2010), DOI: $10.1016 /$ j.cbi.2010.06.014

44. S.P. Slight, R. Howard, M. Ghaleb, N. Barber, B.D. Franklin, and A.J. Avery, British Journal of General Practice, 63(615), e713(2013), DOI:10.3399/bjgp13X673739

45. A. Saleem, M.A. Farooq, M.M. Ali, F. Qureshi, and A. Amin,. International Current Pharmaceutical Journal, 5(12), 118(2016), DOI:10.3329/icpj.v5i12.30414

46. J. Pedraza-Chaverrí, L.M. Reyes-Fermín, E.G. Nolasco-Amaya, M. Orozco-Ibarra, O.N. MedinaCampos, O. González-Cuahutencos, I. Rivero-Cruz, and R. Mata, Experimental and Toxicologic Pathology, 61(5), 491(2009), DOI:10.1016/j.etp.2008.11.002

47. C. Oostenbrink, A. Villa, A.E. Mark, and W.F. Van Gunsteren, Journal of Computational Chemistry, 25(13), 1656(2004), DOI:10.1002/jcc.20090

48. A.P. Terzyk, Journal of Colloid and Interface Science, 275(1), 9(2004), DOI: $10.1016 /$ j.jcis.2004.02.011

49. V.C. Jordan, and M. Morrow, Endocrine Reviews, 20(3), 253(1999), DOI:10.1210/edrv.20.3.0368

50. A.D. Buckingham, Philosophical Transactions of the Royal Society of London. B, Biological Sciences, 272(915), 5(1975), DOI:10.1098/rstb.1975.0066

[RJC-5770/2020] 